\title{
Escuela nueva y antropología aplicada: la educación rural en el Perú en las décadas de 1920 y 1930
}

\section{Mercedes Giesecke}

Universidad Nacional Mayor de San Marcos

Se examina la conexión entre la escuela nueva y la antropología aplicada a través del pragmatismo clásico estadounidense, así como sus efectos en las reflexiones sobre el problema del indio, la difusión de métodos de la escuela nueva y las propuestas para la educación rural en el Perú.

La nueva educación, o escuela nueva, es una tendencia que fue entendida como una propuesta para implementar la educación popular —e incluso la educación universitaria —, vinculando la educación con la comunidad, su historia y geografia.

La bibliografia revisada pone énfasis en el desarrollo de la ciudadanía a través de la educación, sobre todo en el ámbito rural. Considera un texto sobre las mutuas influencias entre Boas y Dewey, así como la reflexión sobre la educación del indio a través de doce autores peruanos. Asimismo, diecisiete boletines de la Unión Panamericana sobre aspectos de los métodos educativos entre 1927 y 1931. Finalmente, cuatro publicaciones del Ministerio de Educación Pública entre 1938 y 1939, que formulan las políticas orientadas a la educación rural.

Palabras clave: escuela nueva, antropología aplicada, cambio social, educación popular e indígena en el Perú en la década de 1930, educación rural. 


\title{
New School and Applied Anthropology: Rural Education in Peru in the 20s and 30s
}

\author{
ABSTRACT
}

The connection between the new school and applied anthropology through classical American pragmatism is examined here, as well as its effects on the reflections on the Indian problem, the spread of the new school methods, and on proposals for rural education in Peru. New education or new school is a trend proposed to implement popular education and even university education, linking education to the community, its history and geography.

The literature reviewed emphasizes the development of citizenship through education, especially in rural areas. It considers a text on the mutual influences between Boas and Dewey, twelve Peruvian authors on Indian education, a resume of seventeen newsletters of the Pan American Union on aspects of educational methods, between 1927 and 1931, as well as four publications of the Ministry of Education between 1938 and 1939, which formulate policies to rural education.

Keywords: new school, applied anthropology, social change, popular and indigenous education in Peru in the thirties, rural education. 
Este artículo revisa la importancia histórica de la educación como estrategia para formar ciudadanía dentro de sociedades multiculturales como el Perú entre las décadas de 1920 y 1930. La definición de educación como estrategia está significativamente sustentada en el enfoque pragmático progresivo de la filosofía y de la antropología, así como del conjunto de las ciencias aplicadas con la finalidad de crear bienestar social.

Dewey (1859-1952) y Boas (1858-1942) fueron aliados intelectuales y políticos por casi cuarenta años, entre los años 1904 y 1942. Estudiaron juntos en la Universidad de Chicago y ambos trabajaron en la Universidad de Columbia. Fueron años muy productivos en los que elaboraron, de manera colaborativa, los conceptos de cultura y naturaleza que luego transmitieron a sus alumnos. Como señalan Torres y Hobbes (2015, p. 154), ambos intelectuales tuvieron una misma formación «académica progresiva».

Por un lado, la filosofía de Dewey ha sido importante para correlacionar los contextos socioculturales con los tipos ideales de humanidad. Ello le resultó útil para determinar que la investigación filosófica se puede desarrollar dentro del contexto de la experiencia particular de los grupos humanos, para propiciar el progreso social desde un marco de referencia pedagógico que sustentaba el cambio social y la acción política.

Por su parte, Boas estuvo preocupado por traducir las cuestiones antropológicas a cuestiones sociopolíticas. En este sentido, la creación de su concepto de cultura, basado en la experiencia etnográfica, le sirvió para sostener y oponerse a las ideas hegemónicas de la inferioridad de los pueblos no occidentales, así como para cuestionar la validez del concepto «raza» como categoría para explicar las diferencias culturales.

Ambos consideraron que el particularismo histórico y geográfico era fundamental para comprender la diversidad cultural. Dewey entendió esta diversidad cultural como núcleo para la investigación filosófica originada en la experiencia 
de los pueblos, marco de sus saberes y conocimientos. Boas contribuyó con Dewey al elaborar el concepto de cultura a partir de la investigación etnográfica, mostrando que cada pueblo elaboraba su cultura como respuesta a la adaptación. Tanto para Boas como para Dewey, la experiencia ocupa un lugar estratégico:

[...] había un imperativo científico en registrar cuidadosamente la experiencia de los diferentes pueblos alrededor del mundo. El desarrollo de la definición de la cultura para Boas trajo orden metodológico para la comprensión de las experiencias significativas en diferentes sociedades [...] Para Dewey y gran parte de la filosofía americana «clásica», hubo una reconceptualización del punto de partida para la investigación, es decir, que debería ser la experiencia vivida en sí misma. A partir de esta perspectiva fenomenológica [...] ambos estaban insistiendo en la primacía de la experiencia en sus respectivas disciplinas y rechazaron los enfoques jerárquicos de la investigación (Torres y Hobbs, p. 140).

La íntima relación entre cultura y naturaleza fue reflexionada desde el pragmatismo de Dewey y James (1842-1910), quienes «aplicaron el concepto de la tendencia de los organismos para adaptarse a la naturaleza, a la tendencia de los seres humanos para adaptarse a la historia de su cultura, así como al comportamiento de otros individuos» (Torres y Hobbs, 2015, pp. 140-141). Asumieron que la experiencia era la forma en que los seres humanos probaron y seleccionaron las conductas que «daban lugar a resultados positivos». A ello se sumó el pensamiento de Boas: «[...] la tendencia de la gente a hacer cosas, de actuar para desarrollar nuevas actividades sin diseño consciente o conciencia (vigilante), para luego inventar racionalizaciones secundarias originadas después de ocurridos los hechos...» (ibid.). Hubo una convergencia entre el pensamiento de Boas y el de Dewey:

Dewey estuvo influenciado por Boas y por los principios de la antropología americana, pues hizo el primer intento de comprender la naturaleza desde la perspectiva de una antropología moderna [...] Boas ayudó a desarrollar el concepto de cultura, que desempeñó un papel clave en el desarrollo de la antropología cultural estadounidense y la comprensión de la experiencia hecha por Dewey $[\ldots]$ Fue un instrumento en el desarrollo de un concepto antropológico de la naturaleza (ibíd., p. 142).

Boas publicó una definición explícita de la cultura en 1930: «[...] todas las manifestaciones de los hábitos sociales de una comunidad, las reacciones de los individuos afectados por los hábitos del grupo en el que vive, los productos de las actividades humanas determinadas por esos hábitos» (Torres y Hobbs, 2015, 
p. 144), y creó una noción moderna relativista de cultura cuando pasó «del uso de su forma singular a su forma plural»:

[...] universalmente experimental porque asumió que cada sociedad tenía cultura políticamente progresiva porque [...] desafiaba las asunciones occidentales acerca de la carencia de civilización, de un desarrollo inferior, de los pueblos no occidentales [...] Boas estuvo especialmente atento a la participación de los individuos en los procesos culturales (Torres y Hobbs, 2015, p. 145).

Además, comprendió la mutua influencia entre la historia colectiva, con sus entornos contextualizados y el individuo.

Boas, en un artículo de 1920, en Los métodos de la etnología, describió el problema de la comprensión de la relación individuo-sociedad como «una de las más importantes que deben abordarse en un estudio de los cambios culturales $[\ldots]$ la experiencia humana culturalmente universal es colectiva, contextualmente dependiente e inclusiva del pensamiento individual y la comunicación [...] parte integral de la obra de Dewey» (Torres y Hobbs, 2015, p. 145).

Boas puso mucha atención al individuo (Torres y Hobbs, 2015, p. 145), lo que tuvo un gran impacto en la política progresiva y en la democracia liberal - porque consideró que el individuo podía tener la capacidad de mantener la cultura y también podía cambiar sus formas-, en la naturaleza e importancia del trabajo de campo etnográfico, y porque fue la piedra angular de la metodología de la antropología americana inicial.

Fue esta capacidad de Boas y Dewey para comprender las diferencias individuales a través de un prisma cultural lo que solidificó la base intelectual sobre la que ambos hombres basaron sus convocatorias públicas de justicia social. [...] Dewey y Boas estarían de acuerdo [...] las diferencias en el logro de la «civilización» por las respectivas «razas» eran problemas para resolver, o al menos mejorar, en lugar de ser meramente aceptadas en términos de nociones biológicas de inmutables diferencias raciales (Torres y Hobbs, 2015, p. 145).

Por un lado, Boas fue geógrafo y físico, había «estudiado y teorizado acerca de la influencia de la geografía y el ambiente en la cultura». Por otro lado, una vez que Boas ancló el concepto de cultura en la experiencia, Dewey avanzó «en la teoría de la naturaleza que fuese inclusiva de la cultura como un signo de la experiencia humana» (Torres y Hobbs, 2015, p. 146). 
[Dewey] argumenta que la experiencia y el conocimiento son perfectamente ocurrencias naturales dentro de un mundo en el que intentamos gestionar nuestros asuntos y resolver situaciones problemáticas [...] sostenía que significados y valores son realidades de origen natural. Significados y valores ${ }^{1}$ se dan en nuestra experiencia; por lo tanto, son naturales (Torres y Hobbs, 2015, p. 147).

Para concluir, el aporte del enfoque pragmático a la comprensión del sentido de pertenencia del hombre o humanidad con la naturaleza necesita de la mediación de la experiencia ${ }^{2}$. Ello solo es posible a través de experiencias significativas, que ocurren únicamente por medio de la comunicación, definida como:

[...] una inteligencia transaccional que hace que las experiencias más significativas ocurran naturalmente y que son relacionadas con otras experiencias [...] La comunicación es un «multiplicador» [...] En las propias palabras de Dewey, la comunicación se define como «[...] el establecimiento de la cooperación en una actividad en la que no son socios, y en la que se modifica la actividad de cada uno al ser regulados por la asociación». Como tal, es más que simplemente proporcionar expresión a lo que ya existe. Dicha participación compartida aumenta la percepción, el motivo del por qué la teoría de la comunicación de Dewey está estrechamente relacionada con su filosofía social y política, según la cual la democracia es fundamentalmente un ideal moral en lugar de ser simplemente una cierta comprensión particular de la esencia de, o papel primordial para gobernar (ibíd., p. 151).

La teoría antes reseñada formó parte de una amplia corriente de pensamiento orientada a crear condiciones de participación ciudadana para miles de indígenas en el mundo y estuvo conectada con las ciencias humanas y sociales, que se incluyeron en el pensamiento pedagógico de la época, afín a la educación activa o escuela nueva. El enfoque etnográfico ha sido central en la comprensión del

1 «Este humanismo, naturalismo cultural era un tema unificador que recorrió a través del desarrollo filosófico de Dewey [...] la experiencia de Dewey significa "nuestra convivencia cultural compartida del mundo" o "[...] nuestra compartida y encarnada vida simbólica, son las formas significativas con las que habitamos el mundo". Esta concepción antropológica de la experiencia constituye un desafío a la establecida oposición dualista de la mente y la naturaleza, al menos en la modernidad, hecha por Descartes» (ibid., p. 148).

2 De esta manera, la comunicación es un arte de hacer del presente más significativo, un énfasis particularmente evidente en las partes posteriores de la naturaleza humana y conducta. Para Dewey, nada de esto ocurriría fuera de, o de alguna manera independiente, de la naturaleza. Todo es perfectamente natural y al mismo tiempo cultural, es decir, es radicalmente experimental (ibid., p. 153). 
modo de ser de las personas en función de su cultura. El Perú también hizo eco de estas grandes innovaciones pedagógicas.

El siguiente acápite muestra un conjunto de autores peruanos que han escrito sobre el problema indígena a la luz del enfoque progresivo pragmático anteriormente planteado por Dewey y Boas. Estos autores peruanos fundamentaron un nuevo ideal de persona y sociedad, una nueva teoría que permitiera impulsar el cambio general de la sociedad peruana, sobre todo enfocándose en el problema indígena, asumido como un tema de educación de la persona.

Los autores peruanos que abordaron la reflexión sobre el tema de la educación del indígena fueron académicos de la Universidad San Marcos como Luis Miró Quesada, de la Universidad San Antonio Abad del Cusco, y Luis E. Valcárcel, quien escribió para Amauta, además de ser decano de Letras de San Marcos y ministro de Educación, junto a otros escritores cusqueños como Víctor Guevara, Carlos Arbulú M. y Atilio Sivirichi, y Rafael Larco Herrera, quien estudió agronomía en la Universidad de Cornell. Todos ellos escribieron sobre la educación indígena para la Revista Universitaria del Cuzco. Asimismo, normalistas como Enrique Guzmán y Valle, quien fuera director de la Escuela Normal de Varones de Lima; Carlos Velásquez, quien escribió en Amauta; Elías Ponce Rodríguez, quien estudió en San Marcos y escribió en Amauta; José Antonio Encinas, quien estudió y luego fue director de la Escuela Normal de Varones de Lima y en San Marcos, donde estudió y luego fue su rector; los profesores trujillanos César Acurio y María Judith Arias quienes escribieron en Amauta, y el profesor ayacuchano Moisés Cavero, entre muchos otros.

Estos autores escribieron entre los años 1909 y 1939, pero sobre todo se concentraron en el segundo lustro de la década de 1920, entre 1926 y 1933 . Se presentan a continuación los principales rasgos de su definición antropológica, imbuida en un concepto holístico que muestra la íntima identificación de la persona con el todo que la constituye. Los argumentos de los normalistas y profesores giran en torno a la pedagogía a partir del sustento científico del conocimiento de la «naturaleza biopsíquica» del niño (Guzmán y Valle, 1921, p. 2), tal como se muestra en el pensamiento de varios de ellos:

«La misión principal de la escuela es desarrollar al máximo la personalidad del niño, basándose en las fuerzas interiores perfectamente disciplinadas. Cambia el papel del maestro de ser únicamente transmisor pasivo de conocimientos, a ser un "director biológico" de la escuela. Se interesa por "los problemas relativos de la vida del niño en su cuádruple aspecto social, mental, físico y psíquico"» (Encinas, 1938, p. 15). «La educación es una función social, 
luego debe corresponder a las condiciones biológicas y sico-sociológicas del medio» (Acurio y Arias, 1929, p. 27). «La experiencia adquiere una gran importancia en las ciencias educativas» (Ponce, 1930, p. 7). «La pedagogía, por tanto, "debe tener un fundamento biológico; psicológico; sociológico; económico; idealista o ético filosófico”» (Velásquez, 1926, p. 26).

Los profesionales universitarios de la UNSAAC argumentaron la importancia de dar a conocer el particularismo histórico que era el origen de la vida cultural del pueblo indígena, como en el caso de Víctor Guevara, quien definió y describió la herencia familiar indígena reconociendo los valores de la familia indígena en cuanto a la estabilidad y al trabajo colectivo. El marco teórico dado por la definición de cultura y naturaleza cobró realce:

«Las culturas sometidas a las leyes generales de la vida; nacimiento, desarrollo y muerte [...] Las culturas en el mundo espiritual son las creaciones máximas de cuya energía se nutren pueblos e individuos [...] Cada personalidad, cada grupo, nace dentro de una cultura y solo puede vivir dentro de ella, como el pez en el agua [...] Vamos por la tierra con nuestro propio mundo a cuestas; conocemos, pensamos, sentimos según el conocer, el pensar y el sentir de la propia cultura [...] Somos hijos, es decir, herederos de un ser que la Naturaleza y la Cultura han formado. La generación espontánea, la mutación, la vida sin historia repugnan, pues, a nuestra mente» (Valcárcel, 1927, p. 2). «La herencia moldea los caracteres inconfundibles de tipo étnico y de unidad social. [...] Enfocan la modalidad subjetiva del indígena» (Arbulú, 1927, p. 45). «La personalidad americana, surge pujante una cultura americana y esta personalidad y esta cultura son netamente indianas o indigenistas» (Sivirichi, 1937, p. 3). «A través del conocimiento de su capacidad, herencia, costumbres, clima» (Larco, 1939, p. 39).

La moral pública, social, familiar e individual se constituyó en un importante motor para la acción reformista, según los normalistas y profesores:

«El problema central es espiritual» (Guzmán y Valle, 1921, p. 2). «Educar es en último término orientar la evolución colectiva en un camino de mejoramiento, sin quitar la personalidad propia del sujeto» (Acurio y Arias, 1929, p. 27). «Educar es [...] despertar la simpatía que nace del conocimiento de la vida del aborigen» (Ponce, 1930, p. 7).

Según los profesionales universitarios:

La moralidad [es la] base de toda democracia [...] La misión de las universidades: formar el ideal nacional» (Miró Quesada, 1926, p. 6). «Lo ideal es formar un nacionalismo constructivo para el Perú y el germinar de una cul- 
tura americana [...] exaltando las glorias del pasado consigue mostrar a las generaciones nuevas el orgullo del pretérito, como expresión del poderío y de fuerza» (Sivirichi, 1937, p. 18). «La inmoralidad [...] es el gran problema que el país tiene que resolver para asimilarlo a su actividad civil» (Larco, 1939, p. 45). «También se propone un mayor control del uso del ingreso regional del Cusco, con la finalidad de garantizar su inversión en la educación pública» (Valcárcel, 1916, p. 3).

La ciencia y la moral se consideraban complementarias: la ciencia debía servir a un bien moral. Según los normalistas y profesores:

«El remedio está en la ciencia y en la escuela» (Guzmán y Valle, 1921, p. 2). «Para educar al niño hay que entrar muy dentro del yo infantil, para afianzar su personalidad. La nueva educación gira alrededor de los intereses del niño» (Velásquez, 1926, p. 25). «Conocer al niño indígena [...] es decir, a las costumbres, creencias, tradición, organización social, idioma, condiciones étnicas y aspiraciones de raza» (Acurio y Arias, 1929, p. 27). «La exploración de la mentalidad del indio, comparada con la del habitante de la costa y con la del de la montaña» (Ponce, 1930, pp. 8-9). «Se propone crear escuelas normales para preparar en su seno a los jóvenes indios más aptos para que pudieran ser ellos, en futuro no lejano, los educadores de los demás de su raza [...] En la primera [etapa] se debe enseñar á leer y escribir en el idioma del indio, hasta que él esté suficientemente preparado para que pueda usar el sistema gráfico con verdadera eficiencia. Entonces debe comenzar la segunda etapa, la cual consistiría en el aprendizaje del castellano» (Cavero, 1933, p. 29).

Para poder formar educadores profesionales universitarios, era necesario traer especialistas en metodología, en psicología experimental, para establecer un laboratorio experimental (Miró Quesada, 1926, p. 41). A continuación presentamos los diversos tipos de problemas a resolver en la vida indígena.

Según los normalistas

«Se debía lograr la identificación del maestro con el indígena» (Guzmán y Valle, 1921, p. 3). «Que la escuela sea la gran propagadora de la justicia entre los pueblos» (Velásquez, 1926, p. 26). «Respetar la personalidad indígena: su herencia biológica, su influencia social y la acción nociva del elemento mestizo» (Acurio y Arias, 1929, p. 22). «Se debía hacer el estudio de su constitución social [del indio]: modalidades propias de vida y organización. Estudio de la organización de la propiedad, del trabajo y de las utilidades percibidas por el indio. Investigación sobre las lenguas aborígenes. Estudio de las enfermedades, de los alimentos y de la habitación de los indios» (Ponce, 1930, p. 8). «[Que] la sociedad debe de considerar como función propia no 
solo la de colaborar con la escuela, sino de sustraerse de prejuicios políticos, religiosos y económicos [...] Una formación integral requiere la acción de todos los agentes sociales» (Encinas, 1938, p. 23).

\section{Según los profesionales universitarios}

«Solucionar los problemas políticos, artísticos, económicos, médicos y de higiene» (Miró Quesada, 1926, p. 17). «En ese momento era necesario suspender a los inspectores de instrucción, que fueron sustituidos por los municipios. La crisis financiera trajo el fracaso de la reforma educativa. Pésimos locales escolares fueron el foco de infecciones y enfermedades» (Valcárcel, 1916, p. 6). «Buscaron la defensa a la vida de la familia indígena de las agresiones de los gamonales. El código civil debería reconocer el matrimonio tradicional indígena» (Guevara, 1927, p. 5). «La acción continua de la agrupación criolla o civilizada controla en cierta manera la libre formación de la personalidad indígena $[. .$.$] estudiándola, preparándola y suscitándola para su pronta trans-$ formación» (Arbulú, 1927, p. 45). «El Cusco como el eje del movimiento, como Capital Arqueológica, Indígena y tradicional de América, busca liquidar el feudalismo colonial y promover la conquista del indio a su derecho a la vida civilizada» (Sivirichi, 1937, p. 17). «La educación fue considerada como una palanca de cambio en la mejora de la vida del pueblo indio [...] pero continúa, en su mayoría, librado a la ignorancia en la miseria, con una marcada fobia por la higiene, entregado en alma y cuerpo al alcohol, dopado por la coca, tentaciones que se confabulan en servicio de la expoliación de ciertas autoridades con los caciques y gamonales» (Larco, 1939, p. 45).

Las reflexiones descritas muestran una búsqueda constante de situar el pensamiento educativo, como un soporte moral que relaciona la pedagogía con las ciencias sociales y su aplicación para la formación de la ciudadanía dentro de la población indígena. En el gráfico siguiente se muestra el devenir del pensamiento progresivo en el Perú, desde la preeminencia de Dewey y Boas, su influencia en educadores peruanos y sus propuestas para la educación del indio, así como el impulso y la difusión que los Boletines de la Unión Panamericana dieron a las diversas experiencias de la pedagogía de la escuela activa en el continente americano, finalizando con las políticas educativas del Ministerio de Educación del Perú. Pero ya desde 1910 se había iniciado la investigación etnográfica en el Perú y su institucionalización se dio con la creación de los institutos de etnografía a mediados de la década de 1940. 


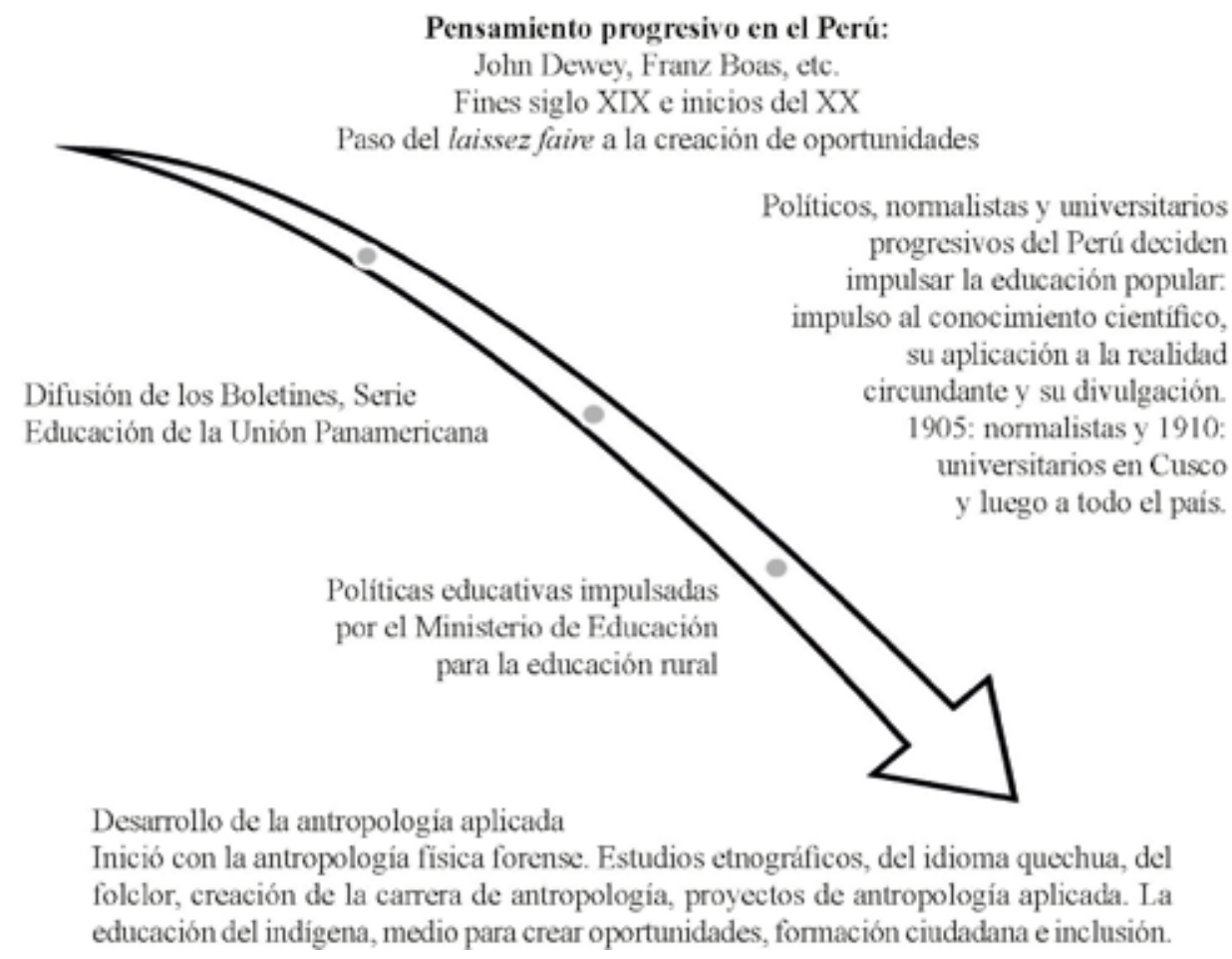

La interacción entre el pensamiento progresivo estadounidense y las experiencias educativas latinoamericanas, con sus contextualizaciones etnográficas, se puede contrastar con la información inscrita en los boletines seleccionados de la Unión Panamericana ${ }^{3}$. Punto de partida: la educación pública en Estados Unidos es gratuita, se tiene una fe general en la educación. El fin de la educación: tener ciudadanos educados para: (i) emprender y dirigir inteligentemente, y (ii) ser trabajadores educados.

La importancia en la presente reseña de los boletines elaborados y distribuidos por la Unión Panamericana consiste en evidenciar una corriente de pensamiento pedagógico que encarnaba un tipo de experiencia educativa dentro de los Estados Unidos y que, a su vez, se fundamentaba en la influencia filosófica y antropológica de Dewey y Boas, respectivamente.

3 Unión Panamericana (o Unión Pan-Americana), organismo dependiente de la Unión de las Repúblicas Americanas creado por resolución de la IV Conferencia de Interamericana 1910 celebrada en Buenos Aires. En abril de 1948 es reemplazado por la OEA. Ver acerca de los boletines en el Anexo 1. 
Los diversos autores consultados dentro de estos boletines, como Amidon, Newlon, Courtis, Parkhurst, Washburn y Smith, fueron representantes de una corriente pedagógica social que expresaba un pensamiento democrático y liberal. Las ciencias naturales y sociales han aportado verdaderamente en el pensamiento de Dewey, quien encarna a la filosofía pragmatista de la educación. Las características de dicho pensamiento, según los autores antes mencionados, buscaron diferenciar la educación tradicional de la progresista. La corriente pedagógica deweyana que llegó al Perú y Callao también lo expresó así. La educación progresista o progresiva considera fundamentales las siguientes ideas:

«Debe ser una expresión vital fructífera, definida como pensamiento creador» (Amidon, 1929, p. 4). «Considera que la educación espiritual es prioritaria a la enseñanza de conocimientos» (Newlon, 1930, pp. 5-6). «La investigación y el experimento son aspectos básicos, porque aprendemos haciendo. La responsabilidad del progreso pasa del maestro al niño» (Amidon, 1929, p. 3). «Considera fundamental «la índole y necesidades del niño para que aumente sus capacidades, para que afronte nuevas situaciones y la diversidad de sus intereses. Se busca integrar la personalidad» (Courtis, 1928, p. 6.). «Pone a la juventud en conexión con la herencia social, y a la vez la educación se convierte en agente de la reconstrucción social. Que los conocimientos se amplíen y se pongan al servicio del hombre» (Callo, 1946, p. 186). «Hoy día hay una creciente demanda por una escuela más realista en sus procedimientos y más sensible a las necesidades individuales y sociales» (Newlon, 1930, p. 13). «La instrucción individual se basa en la filosofía pragmática, que introduce el espíritu de la democracia en la escuela e influye «en el método y organización del material de enseñanza, en el programa de administración de la instrucción, en la exigencia de más libertad» (Courtis, 1928, p. 1). «El plan Dalton trata de socializar la escuela y hacer de ella una comunidad cooperativa. El desarrollar la concentración y fomentar la actividad consciente y la cooperación y al mismo tiempo evitar la instrucción mecánica» (Parkhurst, 1928, sin número de página). «La filosofía fundamental del plan de estudios de Winnetka es que el niño llegue a desarrollar plenamente su individualidad e inculcar en él un sentimiento fundamental de su dependencia y responsabilidad con relación al grupo de que es parte [...] desarrolla la conciencia social [con] una gran variedad de actividades cooperativas en las que cada niño debe subordinar su bien personal al bien público, y también muchas actividades en que cada niño puede desarrollar completamente su propia individualidad (Washburne, 1929, p. 6).

El incipiente desarrollo del sistema educativo en el continente americano, sobre todo en los países latinos, estimuló la idea de que era muy importante promover un mayor impulso a la organización de la educación elemental, que era la 
educación impartida en los primeros dos años de estudios escolares y que era la única educación recibida por muchos. Por este motivo se creía que debía dar las habilidades y conocimientos esenciales para la vida, tales como:

- Ser un buen ciudadano, lo que significaba tener gusto artístico, vivir en dependencia mutua, mantener la relación entre familias e instituciones sociales, conocer procesos industriales y de organización cívica para poder participar en la vida industrial, profesional o cívica; con la formación de hábitos sociales y de estudio, de un elevado carácter ético, con sabio uso del tiempo de ocio.

- Gozar de buena salud. Desarrollar un cuerpo sano y tener un coordinado control muscular.

- Vivir feliz.

- Tener interés por la lectura, aritmética, ortografía, artes gráficas, caligrafía.

Para el caso de la Educación Elemental Rural Agrícola, se consideraba rurales a aquellos lugares con una población de menor de 1000 habitantes, con escuelas unidocentes y pequeñas. Un ejemplo de la influencia del pensamiento educativo progresivo pragmático deweyano es el siguiente de un autor del Boletín:

Windes. En ningún otro campo de las actividades educacionales las condiciones son más caóticas que en el campo de la enseñanza elemental de la agricultura. [Se sugiere] el método del problema práctico, consiguiendo con esto que el interés natural del alumno, considerado como base para la selección de los problemas y condiciones locales, sea el factor dominante en pro o en contra de la materia de que se trata [...] Los objetivos relacionados con la enseñanza vocacional; un desarrollo del sentido de los deberes y privilegios de los miembros de dichos grupos; y la prueba de la aptitud en las distintas vocaciones. [Se busca] la adaptación de los niños al medio ambiente rural, comprendiendo en ellos los asuntos esenciales de la organización social rural y familiarizarse con los medios de comunicación y transporte [...] el amor a la naturaleza [El conocimiento de] la agricultura técnica [El conocimiento del] idioma que contribuye al verdadero conocimiento de la agricultura, incluyendo los materiales esenciales del uso efectivo de la lengua madre como instrumento de expresión [Sugiere] utilizar temas agrícolas como medios de enseñanza para educar a los muchachos y muchachas [...] hacer que el niño se adapte ampliamente, ayudarlo a escoger libre o inteligente la profesión que más le convenga, y, finalmente, suministrarle la enseñanza que lo haga útil y hábil en la profesión que ha escogido $\left(1930, \mathrm{n}^{\circ} 66, \mathrm{p} .1\right)$.

Lo que se buscaba en los boletines era dar un ejemplo de una aplicación práctica de la corriente de pensamiento de la escuela activa a las necesidades de 
aprendizaje de los niños, ya sea en el kindergarten, en la escuela elemental, en la escuela rural agrícola y urbana o en la secundaria.

La escuela progresó en veinte veces la asistencia en cuarenta años en Estados Unidos, entre 1890 y 1930. Crecieron las sumas invertidas en edificios escolares, cursos, métodos y maestros de calidad. Considerando que muchos alumnos pudiesen concluir sus estudios en la escuela elemental, se incidía en la formación vocacional desde temprana edad. También se tomó en cuenta la educación de adultos, así como la necesidad de proporcionar los libros a través de las bibliotecas, para que la información más amplia estuviese al alcance del espíritu creativo de los educandos. Las principales ideas vertidas a través de los boletines otorgan la centralidad al niño, a su individualidad y creatividad, para desarrollar su aprendizaje por medio del motor interno de su propio interés y psicología.

Los cuatro boletines de la Serie sobre Educación que fueron ubicados en los países latinoamericanos se refieren al Perú, Chile, Paraguay y Uruguay. Lo central es valorar, de aquellos años, el compromiso de las políticas públicas con la modernización de la educación, deseando adecuar a la particularidad histórica los métodos experimentales de la educación activa o nueva educación, en beneficio sobre todo de la educación popular y rural, tal como se puede leer a continuación en algunos extractos:

Brainerd. La Nueva Educación se va adaptando a casos locales. El Estado debe garantizar, junto con la sociedad, las facilidades para el desarrollo activo del individuo. También se está adaptando en pueblos indígenas. [En] el Perú hay interés por la «nueva educación». Algunas escuelas visitadas en Arequipa, por ejemplo, están usando los métodos «activos» hasta donde lo permite el material escolar y el programa oficial. La provincia del Cuzco tiene una «escuela ambulante» para indígenas inspirada en las «misiones culturales» de México, que comprende maestros de lectura, escritura y aritmética, agricultura y labores domésticas. En una casa portátil, la escuela permanece durante cuatro meses en cada comunidad rural $\left(1929, \mathrm{n}^{\circ} 49\right.$, p. 11).

Wilson. En Chile actualmente hay dos tipos de escuelas experimentales. Temporales, a cargo de directores extranjeros que trabajan con maestros chilenos y con los niños chilenos [Usa] los métodos Kerschensteiner, Decroly y Dalton. Vineland para los niños de baja mentalidad. Visitadas por maestros de todas partes de Chile. El otro, permanente, bajo la dirección de los mejores educadores chilenos, está dominado por el deseo de asimilar y adoptar toda técnica educativa útil, cualquiera que sea su origen, y al mismo tiempo adaptarla a las necesidades y condiciones chilenas. Permanentes, bajo la dirección de chilenos. En estas escuelas se está desarrollando actualmente la nueva educación del 
nuevo Chile, la que anhelaban y soñaban los educadores y por la cual trabajaban inteligente y ardientemente. La función principal de estas escuelas consiste en someter a mayor experimentación todo lo que parece ser útil de los métodos y los planes educativos extranjeros [...] Entre los experimentos permanentes están las escuelas para adultos, para una comunidad rural, una escuela hogar para los desamparados, una escuela al aire libre para los anémicos o tuberculosos, y la Escuela Experimental Urbana de Santiago. Escuelas Secundarias Experimentales [...] hincapié en la educación física [...] se practicará en excursiones, en trabajos de jardín, en clases al aire libre $\left(1930, n^{\circ} 67\right.$, p. 4).

Cardoso. En el Paraguay, las escuelas diurnas están clasificadas en inferiores, medias y superiores. Las inferiores son rurales y las otras urbanas. Las rurales tienen por misión preparar al hombre rural, al campesino, al agricultor, proporcionándole la enseñanza necesaria para desenvolverse en el ambiente en que debe vivir; imprimiéndole cierto dinamismo para la explotación más racional de la tierra; inculcándole nociones de higiene para evitar los males físicos que aquejan a los hombres de campo. Y las urbanas educan al niño del pueblo dentro de las necesidades del ambiente [...] La orientación de la enseñanza es netamente práctica; tanto en las escuelas rurales como en las urbanas se aplica el método activo conforme a los últimos adelantos de la pedagogía contemporánea. Sin ser descuidada la cultura intelectual, sentimental y volitiva, se da mucha importancia a la enseñanza de los trabajos manuales. En las escuelas rurales se cultiva la tierra, se cuidan animales y se industrializan las materias primas nacionales. Una escuela rural tipo es aquella en que la institución escolar dispone de cuatro o más hectáreas de terreno divididas en chacra, parque, campo de deportes y puestos para vaca, gallinas, puercos y abejas, y en la que los niños fabrican y construyen sombreros, cestos, hamacas; manejan el telar para tejer ponchos, frazadas, jergas, etc. (1929, $\mathrm{n}^{\circ} 14$, pp. 1-2).

Brainerd. Al viajero que dedica algún tiempo a las instituciones educativas del Uruguay le sorprende la excelencia de la enseñanza y la buena preparación de los profesores. A causa del pequeño tamaño de la República y del carácter homogéneo de sus habitantes ha sido posible realizar admirables progresos educativos, que los educadores uruguayos generalmente atribuyen en parte a las ideas llevadas de los Estados Unidos, hacia 1870, por José Pedro Varela [...] En todo el país hay maestros que estudian, discuten y ensayan las nuevas ideas, para lo cual gozan de bastante libertad. El método Decroly ha despertado considerable interés y se está ensayando en diferentes departamentos, así como también en dos escuelas experimentales, cerca de Montevideo, que han recibido recientemente del Congreso una cantidad especial para que puedan realizar más fácilmente el experimento. Para mantener las escuelas públicas al día el Consejo Nacional de Enseñanza Primaria y Normal envía todos los años al extranjero a varios inspectores o directores de escuela en viaje de estudio y observación $\left(1929, \mathrm{n}^{\circ} 51, \mathrm{p} .1\right)$. 
Se buscó comparar los datos anteriores con cuatro documentos del Ministerio de Educación Pública del Perú, que muestran el impulso y entusiasmo que hubo por la educación activa o nueva educación como también se le llamó. El documento de 1938 indica las características de la educación secundaria. Llama la atención el programa de Ciencias Domésticas para el tercer año orientado al aprendizaje de la crianza de animales de corral, jardinería, horticultura y fruticultura, en un nivel general y no solo rural. La reforma planteó:

Que ha llegado el momento de llevar a debido efecto la reforma integral de los planes de estudios y programas de instrucción primaria y secundaria, a fin de amoldarlos a las necesidades de la educación nacional, a los progresos de la pedagogía moderna y a la capacidad mental de los alumnos (Ministerio de Educación Pública, 1939, p. 70). Que los programas vigentes, por demasiado extensos y analíticos, restan tiempo para las investigaciones individuales y colectivas que deben realizar los alumnos en bibliotecas, museos, gabinetes, talleres, campos de experimentación agrícola, etc., así como privan de libertad de acción e iniciática a los maestros para encaminar su labor en el sentido indicado (p. 71). Se crearon escuelas rurales en términos pedagógicos y vocacionales, se les otorgó el presupuesto necesario para su funcionamiento. Se trataba de una propuesta pionera, «dejar en libertad de iniciativa y de acción a los maestros rurales, para que consultando la realidad ambiental, hagan un esfuerzo de adaptación metodológica, plena de originalidad y de experiencias vividas, que una vez conocidas para la administración, servirán para estructurar, ulteriormente, normas didácticas más realistas y acertadas (Ministerio de Educación Pública, 1939b, p. 10).

El enfoque de la educación activa fue una tendencia vigente en la educación peruana a finales de la década de 1930, poco después de la creación del Ministerio de Educación Pública en el Perú en 1935, cuestión que se ha podido constatar a través de los documentos 38 y 39 consultados para la redacción de este ensayo. Tal como se puede apreciar:

La educación abarca los aspectos moral, físico e intelectual. [...] Nuevos métodos de enseñanza. Los principios y los métodos propugnados por la escuela activa hallan amplia repercusión y suscitan inquietud entre los maestros peruanos. Se ensayan con éxito las diversas modalidades de la Nueva Educación con el propósito de adaptarlas a las características de la niñez peruana [...] Montessori, Decroly, Dalton, Proyectos, etc. Los educadores peruanos están cada vez más convencidos de la urgencia de hacer del niño el centro de todas las actividades educacionales (Ministerio de Educación Pública, 1939, pp. 14-15). 
La reforma educativa incluía el interés desde los jardines de la infancia; en aquellos años fueron muy pocos y eran considerados muy necesarios. Se ofrece información sobre la creación de prescolares desde 1931 en Contumazá e Iquitos, así como:

Escuelas privadas oficiales Montessori. Clases al aire libre para el contacto íntimo con la naturaleza. Secciones Froebelianas para la instrucción sensorial. Clase preparatoria anexa a las escuelas elementales con programas especiales de hábitos de higiene personal, intelectual y manual (Ministerio de Educación Pública, 1939, pp. 9-10).

En cuanto a la escuela primaria general o común, eran urbanas, rurales y vocacionales. Su fin era ejercitar la ciudadanía y perfeccionar los conocimientos elementales. Su plan incluía la lengua nacional; es decir, el castellano, el cálculo, historia del Perú, geografía del Perú, higiene, juegos educativos, cantos escolares y patrióticos. Las escuelas vocacionales tuvieron como fin fomentar actividades productivas de la localidad y el estudio de productos naturales e industrias sobre materias primas locales. El maestro tenía la libertad para adecuarse al medio. Se crearon 34 escuelas elementales de diversos tipos tales como:

Escuelas de Agricultura y Ganadería. En estas escuelas se suministra generalmente una instrucción mixta, es decir, la instrucción Primaria Elemental común y los cursos prácticos de Agricultura y Ganadería y sus derivados [...] Hubiéramos querido dar distinta modalidad a su estructura, de acuerdo con las orientaciones de la nueva pedagogía. Pero hemos juzgado más eficaz dejar en libertad de iniciativa y de acción a los maestros rurales, para que consultando la realidad ambiental, hagan un esfuerzo de adaptación metodológica, plena de originalidad y de experiencias vividas, que una vez conocidas para la administración, servirán para estructurar, ulteriormente, normas didácticas más realistas y acertadas (Ministerio de Educación Pública, 1939, pp. 19-20).

Escuelas Industriales y de Artes y Oficios. En Lima funciona el Centro Industrial de Santa Liberata para varones y comprende los siguientes departamentos: Carpintería, con secciones de ebanistería y tallado, Electricidad y Mecánica, Motores de Explosión y Soldadura Autógena y Bellas Artes. Además se enseña Dibujo Profesional y Natural e Higiene Industrial (p. 20).

Escuelas Nocturnas Vespertinas y de Comercio. Formación de auxiliares capacitados para servir en oficinas industriales y comerciales (p. 21).

En conclusión, la idea central sobre la correlación entre antropología aplicada y escuela nueva fue fundamentada, desde que es la cultura en su contexto histórico y geográfico la que va a tratar de ser comprendida desde la pedagogía y, a 
partir de allí, va a desarrollar su propuesta educativa, relevante a las necesidades de la población.

El pragmatismo educativo progresivo estadounidense sirvió de inspiración tanto para su propia reforma educativa como para la reforma educativa latinoamericana. En el caso peruano se hizo evidente en los escritos de los intelectuales peruanos y del Ministerio de Educación Pública. Tuvo aportes relevantes tanto para la educación rural como para colegios particulares.

Dicho pragmatismo progresivo liberal adoptó una fuerza mayor desde la estrecha colaboración de la antropología con la pedagogía, al estudiar el particularismo histórico de las culturas y su importancia estratégica en la formación del individuo. La libertad y creatividad de los maestros y de los educandos era considerada una pieza clave, sobre todo en la difícil situación de la población rural, por su aislamiento y la casi nula participación de los grupos indígenas en la cuestión de la nación por la diversidad de sus costumbres.

\section{REFERENCIAS BIBLIOGRÁFICAS ${ }^{4}$}

Acurio, César y María Judith Arias (1929. La escuela hogar. Proyecto de un nuevo tipo de escuela Indígena. Amauta, 23, pp. 22-34. Lima.

Alderman, L. R. (1929). La escuela de la oportunidad. Serie sobre Educación, 47. Enero.

Arbulú Miranda, Carlos (1927). El problema del indio. La sierra. Órgano de la Juventud Renovadora Andina, I(6), pp. 43-47. Lima.

Asociación Nacional de Normalistas (1921). La educación nacional y los normalistas del Perú. Lima: Guzmán y Valle Editor. Edición extraordinaria.

Atkins, Blanche E. (1929). Importancia de las excursiones escolares. Serie sobre Educación $\mathrm{n}^{\mathrm{0}}$ 53. Julio.

Boletín de la Unión Panamericana (1927). La educación pública en los Estados Unidos de América. Serie sobre Educación nº 33. Octubre.

4 La Serie sobre Educación corresponde al Boletín de la Unión Panamericana. Washington, D.C.: La Unión Panamericana. Imprenta del Gobierno de los Estados Unidos. Cada boletín incluye el artículo de un solo autor. Todos los boletines fueron ubicados en el archivo del que fuera director general de Instrucción Pública entre 1924 y 1930, doctor Alberto Giesecke. Archivado en el IRA-PUCP. Hago un agradecimiento muy especial a la señorita Bach. En Antropología, a Ana Espejo de los Santos, por su intensa y eficiente labor como mi asistente en la preparación de la información digitalizada y ordenada para la redacción de este artículo. 
Brainerd, Heloise (1929). Nuevas corrientes educativas en Ibero América. Serie sobre Educación $\mathrm{n}^{\circ}$ 49. Marzo.

Brainerd, Heloise (1929). La educación pública en el Uruguay. Serie sobre Educación $\mathrm{n}^{\mathrm{o}}$ 51. Mayo.

Byrd Askew, Sarah (1930). Las bibliotecas circulantes y las escuelas rurales. Serie sobre Educación $\mathrm{n}^{\mathrm{o}}$ 65. Septiembre.

Cardoso, Ramón (1929). La instrucción pública en el Paraguay. Serie sobre Educación $\mathrm{n}^{\circ}$ 14. Agosto.

Cavero, Moisés C. (1933). Sobre la educación del indio. Antara. Revista mensual indigenista, 1, pp. 28-29. Noviembre. Cusco-Perú.

Courtis. S. A. (1928). La instrucción individual. Serie sobre Educación $\mathrm{n}^{\circ}$ 41. Junio.

Curtis Grimshaw, Rusell (1928). El ahorro escolar. Serie sobre Educación $n^{\circ} 38$. Marzo.

Encinas Franco, José Antonio (1938). La educación de nuestros hijos. Santiago de Chile: Ediciones Ercilla.

Fuller Barnard, Eunice (1935). La escuela secundaria de ayer y la de hoy. Serie sobre Educación $\mathrm{n}^{\circ}$ 99. Septiembre.

Guevara J., Víctor (1927). El problema indígena. La sierra. Órgano de la juventud renovadora andina, I(6), pp. 2-5. Junio. 1927. Lima-Perú

Jennings, Judson T. (1929). La biblioteca y la educación del adulto. Serie sobre Educación $\mathrm{n}^{\circ} 57$. Diciembre.

Kitson D., Harry (1928). La orientación profesional. Serie sobre Educación n ${ }^{\text {3 } 39}$. Abril.

Larco Herrera, Rafael (1939). El indio, problema nacional. Revista Universitaria. Órgano de la Universidad del Cuzco. Primer semestre. Tomo I. Año XXVIII, $\mathrm{n}^{\circ}$ $76,38-47$

Lincoln, Edward A. (1931). Agrupación de los alumnos según su habilidad. Serie sobre Educación $\mathrm{n}^{\circ}$ 69. Marzo.

Memoria del inspector de instrucción (1916). Boletín Municipal. Órgano del Concejo Provincial del Cusco, I(2), febrero 29.

Ministerio de Educación Pública (1938). Planes de estudios y programas para el tercer año de Enseñanza Secundaria. Dirección de Educación Pública. Edición Oficial.

Ministerio de Educación Pública (1939). Escuelas Rurales del Perú. Reglamentos, Plan de Estudios, Programas e Instrucciones Generales para su Funcionamiento. Lima: Dirección de Educación Pública.

Ministerio de Educación Pública (1939a). La educación pública en el Perú. Suplemento de la Revista de Educación. Órgano del Ministerio de Educación Pública. Enero. 
Ministerio de Educación Pública (1939b). Legislación Educacional. Compilación cronológica de Leyes, Decretos y Resoluciones correspondientes a los años de 1935 a 1938. Edición oficial. Lima: La Nacional.

Miró Quesada, Luis (1926). Pedagogía universitaria y educación popular. La misión de nuestra Universidad. Lima.

Newlon, Jesse H. (1930). La influencia de John Dewey en las escuelas. Serie sobre Educación n ${ }^{\circ}$ 61. Abril.

Parkhurst, Helen (1928). El Plan Dalton. Serie sobre Educación n ${ }^{\circ} 45$. Noviembre.

Ponce Rodriguez, E. (1930). La educación del indio. Lima: Minerva.

Reeder, Edwin (1929). Una lección con proyecciones. Serie sobre Educación n ${ }^{\circ} 50$. Abril.

Rodgers, Leola (1929). El método de proyectos en las escuelas elementales. Serie sobre Educación n ${ }^{\circ}$ 52. Junio.

Sección Cooperativa de la Unión Panamericana (1930). El sistema cooperativo en la enseñanza industrial. Serie sobre Educación $n^{\circ}$ 58. Enero.

Sivirichi, Atilio. El contenido espiritual del movimiento indígena. Conferencia ofrecida en el Paraninfo de la Universidad del Cuzco, el día 25 de agosto de 1936, Revista Universitaria. Órgano de la Universidad del Cuzco. XXVI(72), pp. 2-23.

Smith, Eugene R. (1931). Una escuela activa en el campo. Del boletín de la Unión Panamericana. Serie sobre Educación $\mathrm{n}^{\circ}$ 68. Febrero.

Torres Colón, Alejandro y Charles Hobbs (2015). The Intertwining of Culture and Nature: Franz Boas, John Dewey, and Deweyan Strands of American Anthropology. Journal of the History of Ideas, 76(1), pp. 139-162. University of Notre Dame y Texas State University. http://dx.doi.org/10.1353/jhi.2015.0002

Valcárcel, Luis E. (1927). Ideario. La sierra. Órgano de la juventud renovadora andina, I(7), pp. 1-5. Julio. Lima-Perú.

Velásquez, Carlos A. (1926). La nueva educación. Amauta, I(2), pp. 25-26. Octubre. Lima.

Washburne, Carleton (1929). La filosofía del Plan de Estudios de Winnetka. Serie sobre Educación $\mathrm{n}^{\circ}$ 48. Febrero.

Wilson, Lucy L. W. (1930). El nuevo programa educativo de Chile. Serie sobre Educación $\mathrm{n}^{\circ} 67$. Noviembre.

Windes. E.E. (1930). Objetivos de la Escuela Rural Agrícola Elemental. Serie sobre Educación $\mathrm{n}^{\circ}$ 66. Octubre. 


\section{ANEXO 1. BOLETINES DE LA UNIÓN PANAMERICANA}

\section{CONTENIDOS: 1. EDUCACIÓN ACTIVA / 2. PROPUESTAS EXPERIMENTALES / 3. DIDÁCTICA Y MÉTODOS}

1. Educación activa: Fuller Barnard, Eunice. La escuela secundaria de ayer y la de hoy. 1935. Boletín de la Unión Panamericana.

La educación pública en los Estados Unidos de América. 1927.

Dewey: Newlon, Jesse H. La influencia de John Dewey en las escuelas. 1930. Intrucción individual: Courtis, S.A. 1928.

Amidon, Beulah. El Kindergarten en los Estados Unidos. 1928.

2. Planes de trabajo experimentales: Plan Dalton. Parkhurst, Helen. En Plan de Educación 1928. Winnetka. Washburne, Carleton. La filosofía del Plan de Estudios de Winnetka. 1929.

Las escuelas «Platoon» de la ciudad de Santa Mónica. 1931.

3. Formación del carácter: Curtis Grimshaw, Rusell. El ahorro escolar. 1928.

Didáctica: Reeder, Edwin. Una lección con proyecciones. 1929. Atkins, Blanche E. 1929. Importancia de las excursiones escolares.

Método de proyectos: Rodgers, Leola. El método de proyectos en las escuelas elementales. 1929.

Bibliotecas: Jennings, Judson T. La biblioteca y la educación del adulto. 1929. Byrd Askew, Sarah. Las bibliotecas circulantes y las escuelas rurales. 1930.

Enseñanza industrial: Sección Cooperativa de la Unión Panamericana. El sistema cooperativo en la enseñanza industrial. 1930. Kitson D, Harry. La orientación profesional. 1928. Alderman, L. R. La escuela de la oportunidad. 1929.

Agrupación según la habilidad: Lincoln, Edward A. Agrupación de los alumnos según su habilidad. 1931.

Escuela rural: Windes. E.E. Objetivos de la escuela rural agrícola elemental. 1930. Smith, Eugene R. Una escuela activa en el campo. 1931. 


\section{EDUCACIÓN PROGRESIVA EN PAÍSES LATINOAMERICANOS}

Perú. Brainerd, Heloise. Nuevas corrientes educativas en Ibero América. 1929. Uruguay. Brainerd, Heloise. La educación pública en el Uruguay. 1929.

Paraguay. Cardoso, Ramón I. La instrucción pública en el Paraguay. 1929.

Chile. Wilson, Lucy L. W. El nuevo programa educativo de Chile. 